\title{
Transfer printed multi-color integrated devices for visible light communication applications
}

\author{
K.Rae $^{1}$, E.Xie ${ }^{1}$, C.Foucher ${ }^{1}$, B.Guilhabert ${ }^{1}$, R.Ferriera ${ }^{1}$, D.Zhu ${ }^{2}$, D.J.Wallis ${ }^{2}$, C.J.Humphreys ${ }^{2}$, R.A.Oliver ${ }^{2}$, \\ E.Gu' ${ }^{1}$, N.Laurand ${ }^{1}$, and M.D.Dawson ${ }^{1}$ \\ ${ }^{I}$ Institute of Photonics, Dept. of Physics, SUPA, University of Strathclyde, Glasgow, UK \\ ${ }^{2}$ Dept. of Materials Science and Metallurgy, University of Cambridge, Cambridge, UK \\ Katherine.rae@strath.ac.uk
}

\begin{abstract}
Integrated multi-color devices for visible light communication applications are fabricated by transfer printing blue-emitting GaN light emitting diodes (LEDs) onto a greenemitting LED array and a colloidal quantum dot color-converter structure.

OCIS codes: 230.3670 light emitting diodes, 160.6000 Semiconductor materials, 060.4510 Optical communications
\end{abstract}

\section{Introduction}

The controlled mechanical integration of photonic structures onto non-native substrates using transfer printing (TP) techniques is paving the way to high-performance, heterogeneous and multi-functional integrated photonic devices. TP allows fully fabricated semiconductor structures to be picked up from their growth substrate with an elastomeric stamp and then printed onto another capability enhancing substrate. The TP technique has already been demonstrated for the fabrication of devices for flexible displays, integrated photonics and bio-sensing [1]-[3].

Using a modified nanolithography system as a means of TP, we have recently reported this technique with printing precision on the nanoscale [4], as well as adhesive-less printing of LEDs onto rigid substrates such as diamond to enhance LED performance [5]. We have also reported TP of nanowire lasers onto various substrates [6].

GaN-based LEDs have been crucial in enabling the development of solid-state lighting thanks to their high power and high efficiency. They also benefit from high modulation bandwidths, making them suitable for visible light communication (VLC) applications [7]. In this work we apply the TP technique to the fabrication of two different multi-color platforms for VLC: first a dual-color GaN-based LED array, and then an integrated ultra-thin LED-color-converter structure.

\section{Fabrication and characterization of multi-color integrated devices}

\section{A. Dual-color integrated LED array}

GaN-based blue- and green-emitting LEDs are ideal for VLC because of their high optical power and high modulation bandwidths. By combining LEDs with two different colors, two separate streams of data can be transmitted at the same time and/or by novel forms of spatial encoding such as Space Shift Keying. The TP technique is advantageous for this as it allows for the implementation of different color LED elements on a single chip with little limitation on the separation of elements.
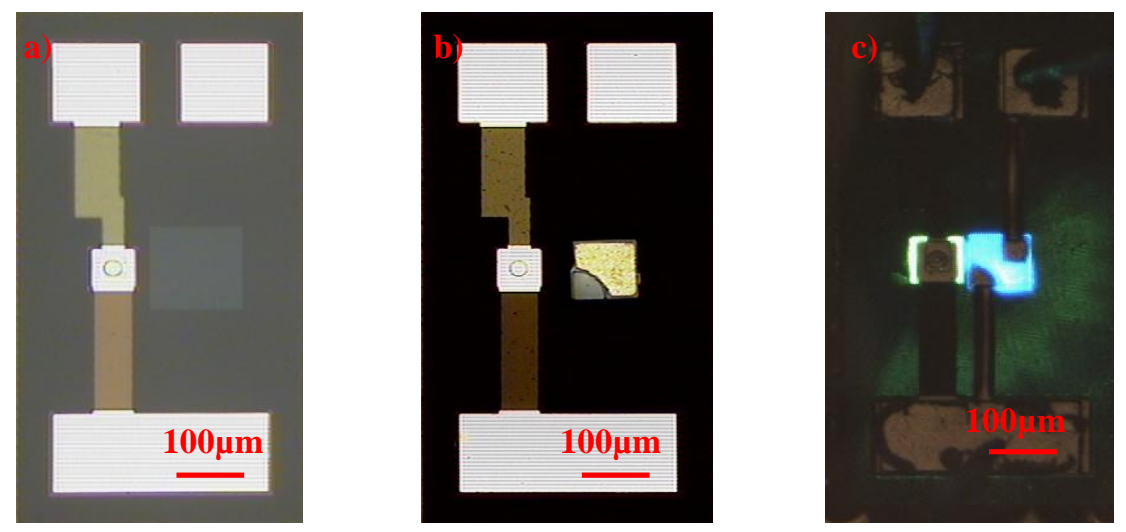

Figure 1: Three main steps in the fabrication of the dual-color LED array: a) First, a green LED element is fabricated (on sapphire), b) then a blue LED from GaN-on-Si is TP next to the green element. c) Finally the LED elements are electrically addressed with Ti/Au metal tracks allowing integrated dual color emission. 
The first steps in the realization of the dual-color integrated LED arrays are the fabrication of the blue and green LED elements separately. The blue elements for TP are fabricated from GaN-on-Si wafers [4,5]; the green ones are fabricated from GaN-on-sapphire, which also serves as the receiving substrate for the printed blue elements. The blue LED elements are then transfer printed onto the green LED chip to create an array of alternating green and blue LED elements. After TP the chip is electrically insulated, with metal tracks deposited to complete the dual-color array. The main steps in this fabrication process can be seen pictorially (plan view optical micrographs) in Figure 1.

Once the dual-color blue-green device is fabricated, the electrical, optical and modulation bandwidth characteristics of the individual LED elements are measured and shown in Figure 2.
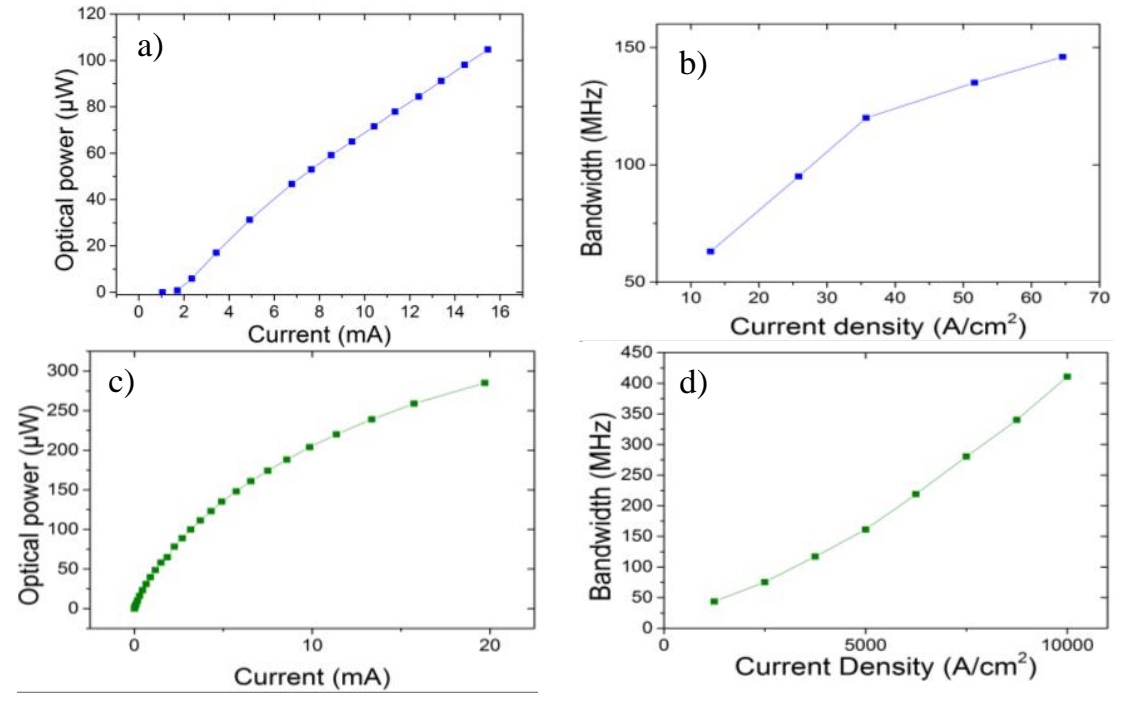

Figure 2: a), c) optical power and b), d) electrical-to-optical modulation bandwidth characteristics of the blue TP, and green LED elements respectively.

As shown, both blue and green LED elements on this dual-color array have comparatively good performance, with the blue element achieving over $0.1 \mathrm{~mW}$ and the green element over $0.25 \mathrm{~mW}$ of optical power at similar currents. The electrical-to-optical modulation bandwidth exceeds $140 \mathrm{MHz}$ and $400 \mathrm{MHz}$ for the blue and green LED elements respectively. These optical power and bandwidth characteristics indicate that this dual-color integrated array is suitable for VLC applications and should achieve data rates of over $1 \mathrm{~Gb} / \mathrm{s}$ when implemented in an appropriate VLC link.

\section{B. Integration of LEDs with CQD color-converter flexible glass platform}

Current visible LED sources make use of down-converting phosphors combined with blue-emitting GaN-based LEDs to efficiently produce light at longer wavelengths or even to produce white light. However, because phosphors have long $(\sim \mu \mathrm{s})$ luminescence lifetimes, they do not achieve the high modulation speeds required for VLC. Colloidal quantum dots (CQDs) are an alternative color-converting medium that is attractive for VLC because they have shorter ( 10ns) fluorescence lifetimes. These higher modulation speeds and narrow emission linewidths make CQDs desirable candidates for implementing wavelength division multiplexing in VLC and/or achieving a wide color gamut for displays.

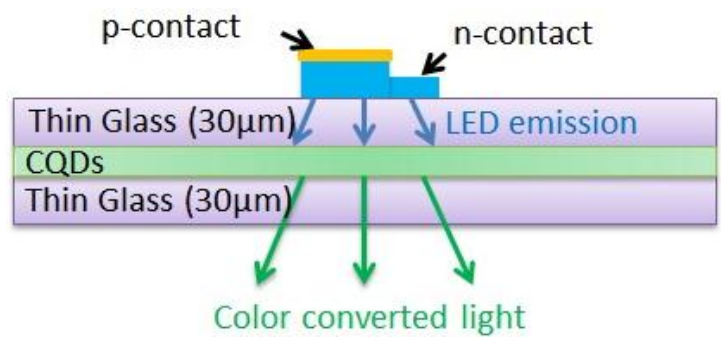

Figure 3: Schematic of the TP LED on the CQD ultra-thin flexible glass color-converter.

We apply the TP technique for integration of GaN-based blue-emitting LEDs with films of CQDs that have been encapsulated between two sheets of ultra-thin $(30 \mu \mathrm{m}$-thick) glass. This gives integrated LED-color-converter 
devices to convert the blue LED light to light at wavelengths from red, orange to green. The blue-emitting TP LEDs (again fabricated from $\mathrm{GaN}$-on-Si) are integrated with these three CQD color-converting flexible glass structures as

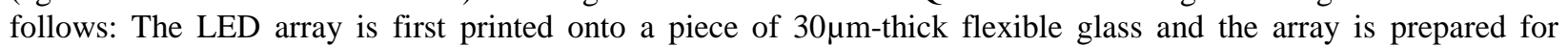
electrical addressing with the deposition of Ti/Au metal tracks. This is then used as the top encapsulation layer for the CQD color-converting film that is formed on a second flexible glass sheet as shown in Figure 3.

The LEDs are thus integrated onto the flexible glass CQD color-converter, enabling LED light to be directly coupled to the CQD region (Figure 4). The absorption of the blue LED light is over $96 \%$ for all three colorconverting samples and forward power conversion efficiencies of $8.8 \%, 15 \%$ and $16 \%$ are obtained, respectively, for the red, orange and green CQD samples. Some light is waveguided in the plane of the CQD film and enhancement of forward conversion efficiency could be obtained by the addition of light extracting features.
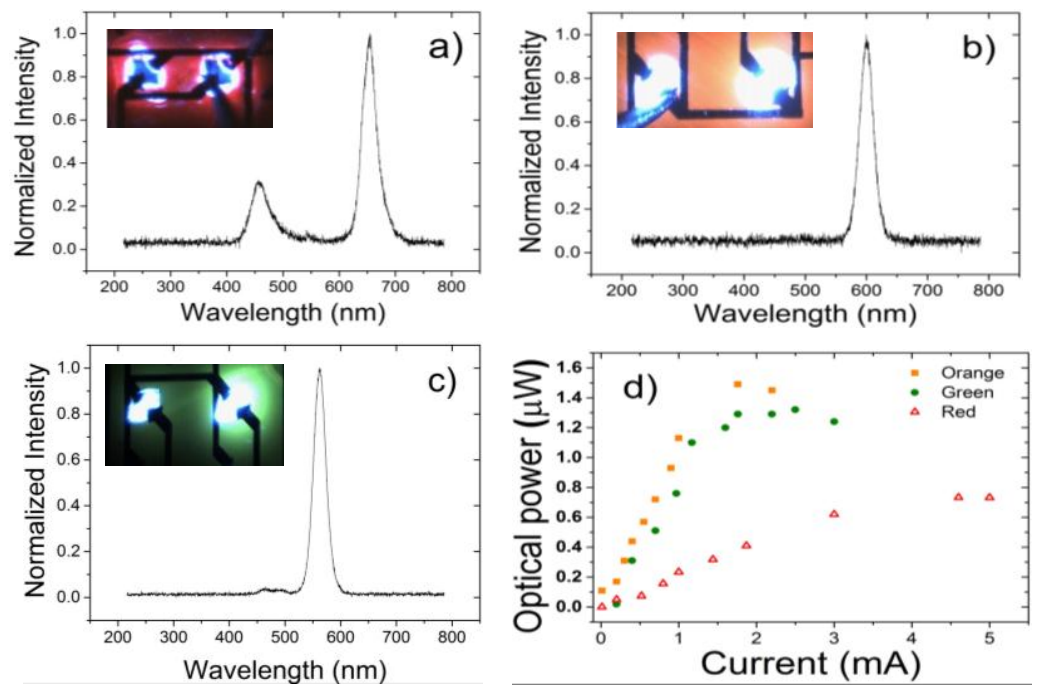

Figure 4: Spectra from the LED printed a) on the red CQD color-converter, $b$ ) the orange CQD color-converter and c) the green CQD color-converter and d) optical power characteristics of all three CQD color-converter devices.

\section{Conclusion}

We have shown the application of transfer printing for fabricating multi-color devices with potential for visible light communications applications. The high modulation bandwidths of LEDs on a dual-color integrated LED device show the potential for combined data rates over $1 \mathrm{~Gb} / \mathrm{s}$ and new routes to spatial modulation. Transfer printed integrated LED-CQD color-converters show efficient conversion of blue LED light to longer wavelengths, and while the low power of these devices may be a limitation in terms of data rate, we will report on the data transmission characteristics of these devices in the future.

\section{References}

[1] T. Kim, K. Cho, E. K. Lee, S. J. Lee, J. Chae, J. W. Kim, D. H. Kim, J. Kwon, G. Amaratunga, S. Y. Lee, B. L. Choi, Y. Kuk, J. M. Kim, and K. Kim, "transfer printing," Nat. Photonics, vol. 5, no. February, pp. 176-182, 2011.

[2] J. Justice, C. Bower, M. Meitl, M. B. Mooney, M. a. Gubbins, and B. Corbett, "Wafer-scale integration of group III-V lasers on silicon using transfer printing of epitaxial layers," Nat. Photonics, vol. 6, no. 9, pp. 612-616, 2012.

[3] D.-H. Kim, R. Ghaffari, N. Lu, and J. a Rogers, "Flexible and stretchable electronics for biointegrated devices.," Annu. Rev. Biomed. Eng., vol. 14, pp. 113-28, 2012.

[4] A. J. Trindade, B. Guilhabert, D. Massoubre, D. Zhu, N. Laurand, E. Gu, I. M. Watson, C. J. Humphreys, and M. D. Dawson, "Nanoscale-accuracy transfer printing of ultra-thin AlInGaN light-emitting diodes onto mechanically flexible substrates," Appl. Phys. Lett., vol. 103, no. 25, 2013.

[5] A. J. Trindade, B. Guilhabert, E. Y. Xie, R. Ferreira, J. J. D. McKendry, D. Zhu, N. Laurand, E. Gu, D. J. Wallis, I. M. Watson, C. J. Humphreys, and M. D. Dawson, "Heterogeneous integration of gallium nitride light-emitting diodes on diamond and silica by transfer printing.," Opt. Express, vol. 23, no. 7, pp. 9329-9338, 2015.

[6] B. Guilhabert, A. Hurtado, D. Jevtics, Q. Gao, H. H. Tan, C. Jagadish, and M. D. Dawson, "Transfer Printing of Semiconductor Nanowires with Lasing Emission for Controllable Nanophotonic Device Fabrication,” ACS Nano, p. acsnano.5b07752, 2016.

[7] J. J. D. McKendry, D. Massoubre, S. Zhang, B. R. Rae, R. P. Green, E. Gu, R. K. Henderson, a. E. Kelly, and M. D. Dawson, "VisibleLight Communications Using a CMOS-Controlled Micro-Light- Emitting-Diode Array,” J. Light. Technol., vol. 30, no. 1, pp. 61-67, 2012.

We gratefully acknowledge Dr A. Kelly and Dr S. Watson for bandwidth measurement support. This work is supported by EPSRC grant EP/K00042X/1. 